\title{
Morphological Stability of Diffusion Couples Under Electric Current
}

\author{
PERRY LEO ${ }^{1,2}$ and ANA RASETTI ${ }^{1,3}$ \\ 1.-Department of Aerospace Engineering and Mechanics, University of Minnesota, Minneapolis, \\ MN 55455, USA. 2.—e-mail: phleo@aem.umn.edu. 3.—e-mail: rasetti@aem.umn.edu
}

We consider the growth and morphological stability of an intermediate phase growing in a binary diffusion couple under electromigration conditions. The growth rate of the intermediate phase depends primarily on the direction of the electromigration current. Current flow that drives the diffusing species enhances growth of the intermediate phase, while current flow in the opposite direction slows growth. The morphological stability of the interfaces between the intermediate phase and the terminal phases depends on the current direction, the relative conductivities of the phases, and the thickness of the intermediate phase. We find that, when the intermediate phase has a higher conductivity than the terminal phases, the current direction that enhances growth of the intermediate phase can also cause an instability. Alternatively, when the conductivity of the intermediate phase is lower than the surrounding phases, the current direction that slows growth can cause an instability. Instability also requires that the thickness of the intermediate phase be larger than some critical value.

Key words: Electromigration, morphological stability, diffusion

\section{INTRODUCTION}

Electromigration arises when an electric current is applied to a metal. It is caused by the interaction between the applied electric field and the positive ions, and the subsequent scattering of these ions and the conduction electrons (wind force). There is also a Coulomb force acting on the ions in the opposite direction to the wind force. The total force can be expressed as $\mathbf{F}_{\mathrm{em}}=|e| z^{*} \mathbf{E}$, where $|e|$ is the electronic unit charge, $\mathbf{E}$ is the applied electric field, and $z^{*}$ is the apparent effective charge. ${ }^{1}$ The total force leads directly to mass transport of ions through the metal.

Electromigration is believed to be the main cause of failure of integrated circuits and microelectronic devices. For example, in metallic interconnects (made of $\mathrm{Al}$ or $\mathrm{Cu}$ ) the order of magnitude of electric current densities- $10^{6} \mathrm{~A} / \mathrm{cm}^{2}$-and the range of temperature at which the device operates $-100^{\circ} \mathrm{C}$ or higher-drives electromigration flux large enough

(Received March 26, 2010; accepted August 13, 2010;

published online September 14, 2010) to cause void nucleation and growth that ultimately leads to an opening in the interconnect. ${ }^{2}$ Electromigration is also important in solder joints, even though the average current densities carried are orders of magnitude lower than in interconnects. This is because the current densities needed to drive electromigration in solder can be much lower than in interconnects, owing to the higher resistivity, effective charge, and diffusivity of solder compared with interconnects. ${ }^{2}$ Electromigration in solder joints leads to excess growth of intermetallic compounds that can cause microcracks to initiate. ${ }^{3}$

In this paper, we consider the electromigrationdriven growth and morphological stability of an intermediate phase growing from two metals in a binary diffusion couple. This work is motivated by the experimental work of Chen and Chen, ${ }^{4-6}$ who describe and measure the growth rate of intermediate phase in $\mathrm{Sn} / \mathrm{Ag}, \mathrm{Sn} / \mathrm{Ni}$, and other systems at different temperatures as a function of applied current. Chen and Chen noticed that the direction of applied current can either enhance or retard the growth of the intermediate phase. Their pictures also show that one of the interfaces between the 
metal and the intermetallic is slightly corrugated, reminiscent of morphological instabilities at planar two-phase interfaces. Motivated by these pictures, we consider the planar growth of an intermediate phase between two metals, as a function of alloy phase diagram, system size, the conductivities of the phases, and the direction and magnitude of the applied current. We then consider small perturbations of the planar interfaces between the intermediate phase and the surrounding metals, and analyze how the applied current will affect their morphological stability.

Morphological stability in electromigrating systems has been studied theoretically by Decuzzi, ${ }^{7}$ Klinger and Levin, ${ }^{8}$ and Klinger et al. ${ }^{9}$ These studies consider interfacial mass flux driven by gradients of electric potential, stress, and curvature along the interface. Stability conditions are found for different system geometries and system parameters such as elastic moduli, current density, and atomic mobility. Maroudas and coworkers have considered similar problems where electric current can stabilize a stress-driven (Asaro-Tiller-Grinfeld) instability. ${ }^{10,11}$ In contrast here we consider bulk mass transport rather than surface diffusion. Orchard and Greer have considered the role of bulk electromigration in the growth of a planar interface in the presence of interfacial reaction barriers. ${ }^{12}$ Their analysis follows Chen and Chen's analyses, as does our unperturbed problem.

In the next section, we set up the problem of intermediate phase growth under an applied electric current, when the interfaces separating the phases are planar. We then consider small perturbations of the interfaces and perform a linear stability analysis. We present and discuss the results and compare them with the experimental results of Chen and Chen. ${ }^{5,6}$

\section{ANALYSIS}

We study the growth of an intermediate phase $\gamma$ between two metals $\alpha$ and $\beta$, when an electric current is applied across the system (Fig. 1). The system is binary, with all phases consisting of the same constituents in different ratios. Specifically we consider an isothermal system at some temperature $T_{0}$ such that the $\alpha$ phase is A rich, the $\beta$ phase is $\mathrm{B}$ rich, and the $\gamma$ phase is an A-B compound. At $T_{0}$ the tie-line compositions of species B are $c_{\alpha}^{\gamma \alpha}$ and $c_{\gamma}^{\gamma \alpha}$ for $\alpha / \gamma$ equilibrium; and $c_{\gamma}^{\beta \gamma}$ and $c_{\beta}^{\beta \gamma}$ for $\gamma / \beta$ equilibrium. We take the $x$-axis (with unit vector $\hat{e}_{x}$ ) along the diffusion couple, and the $y$ - and $z$-axes perpendicular to the couple. The applied electric field or current is along the $\pm x$-direction.

We consider diffusion of species B only. Also we assume that diffusion occurs only in the intermediate $\gamma$ phase and that the compositions in the $\alpha$ and $\beta$ phases are uniform. Hence the composition gradient drives the flow of species B from $\beta$ to $\alpha$ as indicated in Fig. 1. The mass flux of $\mathrm{B}$ in the $\gamma$ phase may be written ss $^{4}$

$$
\mathbf{J}_{\gamma}=-D \nabla c-M c \mathbf{F} .
$$

Here, the first term is mass flux in $\gamma$ owing to bulk diffusion, with diffusivity $D$ and concentration $c$. The second term accounts for the total electromigration flux, with mobility $M$ (related to the diffusivity) and electromigration driving force

$$
\mathbf{F}=|e| z^{*} \mathbf{E}_{\gamma}
$$

where $|e|$ is the unit charge of an electron, $z^{*}$ is the apparent effective charge of the diffusing atoms, and $\mathbf{E}=-\nabla \phi$ is the electric field as found from the electric potential $\phi$. The sign of the electromigration term in Eq. 1 is crucial in determining the current

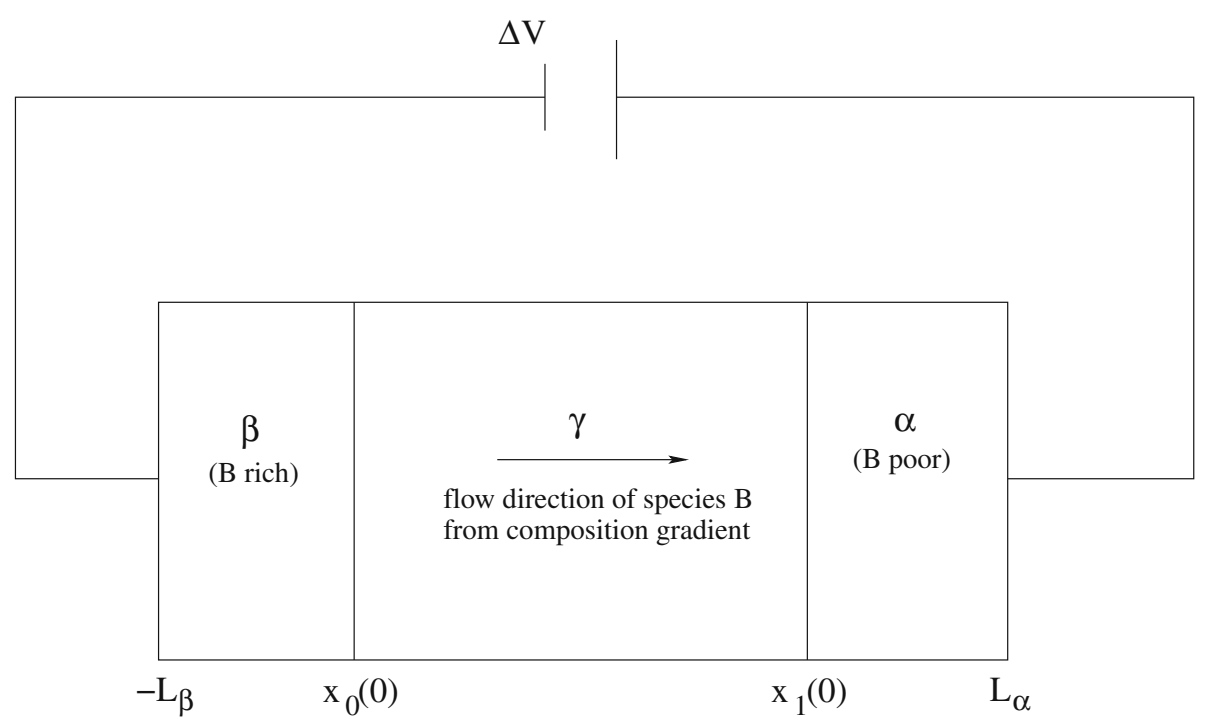

Fig. 1. Schematic of the diffusion couple under electromigration conditions. In the absence of electromigration, flow of solute $B$ in the intermetallic phase $\gamma$ is from the solute-rich $\beta$ phase towards the solute-poor $\alpha$ phase. 
direction that enhances the intermediate phase growth.

We assume that the motion of the interfaces is slow compared with the diffusion speed in the system, and so the flux $\mathbf{J}_{\gamma}$ satisfies mass balance in the the quasistatic limit, $\nabla \cdot \mathbf{J}_{\gamma}=0$. Finally, we take boundary conditions for the composition consistent with the equilibrium phase diagram. We also consider a correction for capillarity consistent with the Gibbs-Thomsen equation (Eq. 21), though our primary focus is on whether there are any circumstances under which current can destabilize an interface.

The electric current $\mathbf{I}=\sigma \mathbf{E}$ where $\sigma$ is conductivity. The electric potentials $\phi$ in each phase must satisfy Laplace's equation together with boundary conditions expressing continuity of both the potential and current:

$$
\begin{aligned}
\phi_{\beta} & =\phi_{\gamma} \\
\sigma_{\beta} \nabla \phi_{\beta} \cdot \hat{n}_{0} & =\sigma_{\gamma} \nabla \phi_{\gamma} \cdot \hat{n}_{0}
\end{aligned}
$$

at the $\beta / \gamma$ interface and

$$
\begin{aligned}
\phi_{\gamma} & =\phi_{\alpha} \\
\sigma_{\gamma} \nabla \phi_{\gamma} \cdot \hat{n}_{1} & =\sigma_{\alpha} \nabla \phi_{\alpha} \cdot \hat{n}_{1}
\end{aligned}
$$

at the $\gamma / \alpha$ interface, where $\hat{n}_{0}$ and $\hat{n}_{1}$ are the unit normals to the $\beta / \gamma$ and $\gamma / \alpha$ interfaces. Finally, because the electric potential satisfies Laplace's equation, we must take a finite system size, and so we specify far-field conditions

$$
\begin{aligned}
\phi_{\beta}\left(-L_{\beta}\right) & =V^{-} \\
\phi_{\alpha}\left(L_{\alpha}\right) & =V^{+} .
\end{aligned}
$$

Because we consider diffusion in $\gamma$ only, we omit subscripts distinguishing the phase in terms related solely to diffusion, though we retain them on quantities associated with the electric field. Also we use a superscript "( 0$)$ " to denote quantities associated with the unperturbed problem, and a superscript "(1)" for quantities associated with the perturbed problem.

\section{Unperturbed Problem}

We first consider planar interfaces, with $x_{0}(t)$ and $x_{1}(t)$ giving the positions at time $t$ of the $\beta / \gamma$ and $\gamma / \alpha$ interfaces, respectively. We choose as the origin the initial position of the unperturbed $\beta / \gamma$ interface, i.e., $x_{0}(0)=0$. For the unperturbed problem, the $\phi_{i}$ are linear in $x$ and so the field can be easily computed. As we only consider mass flow in the $\gamma$ phase, we calculate $\mathrm{d} \phi_{\gamma} / \mathrm{d} x=a_{\gamma}$, with

$$
a_{\gamma}=\frac{\delta_{\alpha} \delta_{\beta} \Delta V}{\delta_{\alpha} L_{\beta}+\delta_{\beta} L_{\alpha}+\delta_{\alpha} x_{0}\left(1-\delta_{\beta}\right)+\delta_{\beta} x_{1}\left(\delta_{\alpha}-1\right)}
$$

and $\Delta V=V^{+}-V^{-}, \delta_{\alpha}=\sigma_{\alpha} / \sigma_{\gamma}$, and $\delta_{\beta}=\sigma_{\beta} / \sigma_{\gamma}$.

We take the composition in the $\alpha$ and $\beta$ phases to be constant, at $c_{\alpha}^{\alpha \gamma}$ and $c_{\beta}^{\gamma \beta}$, respectively. We find the composition field in $\gamma$ by setting $\nabla \cdot \mathbf{J}_{\gamma}=0$ with $\mathbf{J}_{\gamma}$ given by Eq. 1. For the unperturbed problem, $c$ depends on $x$ only, and so this reduces to

$$
\frac{\mathrm{d}^{2} c^{(0)}}{\mathrm{d} x^{2}}-Q \frac{\mathrm{d} c}{\mathrm{~d} x}=0
$$

where

$$
Q=\frac{|e| z^{*} M a_{\gamma}}{D}
$$

as $\mathbf{E}=-\nabla \phi_{\gamma}=-a_{\gamma} \hat{e}_{x}$ and $\nabla^{2} \phi_{\gamma}=0$. The compositions at the planar $\beta / \gamma$ interface and the $\gamma / \alpha$ interface are taken as their equilibrium phase diagram values, i.e., $c_{\gamma}\left(x_{0}\right)=c_{\gamma}^{\beta \gamma}$ and $c_{\gamma}\left(x_{1}\right)=c_{\gamma}^{\gamma \alpha}$.

In the analysis that follows we use $Q$ as our control parameter, that is, the sign and magnitude of $Q$ determine the direction and magnitude of the electric current. In particular note that for the unperturbed problem the flux of solute in the $x$-direction is $J_{\gamma}=-D\left(\frac{\mathrm{d} c}{\mathrm{~d}}-Q c\right)$; that is, when $Q>0$, solute flux from electromigration is from $\beta$ to $\alpha$, as is flux from the concentration gradient (Fig. 1).

By solving Eq. 7 for $c_{\gamma}$ and applying the boundary conditions, we find

$$
c^{(0)}(x)=\frac{\left(c_{\gamma}^{\beta \gamma}-c_{\gamma}^{\gamma \alpha}\right)}{e^{Q x_{0}}-e^{Q x_{1}}} e^{Q x}+\frac{c_{\gamma}^{\gamma \alpha} e^{Q x_{0}}-c_{\gamma}^{\beta \gamma} e^{Q x_{1}}}{e^{Q x_{0}}-e^{Q x_{1}}} .
$$

We find the velocities of the $\beta / \gamma$ and $\gamma / \alpha$ interfaces through the flux balances

$$
\left(c_{\gamma}^{\beta \gamma}-c_{\beta}^{\beta \gamma}\right) \frac{\mathrm{d} x_{0}}{\mathrm{~d} t}=J_{\gamma}
$$

and

$$
\left(c_{\alpha}^{\gamma \alpha}-c_{\gamma}^{\gamma \alpha}\right) \frac{\mathrm{d} x_{1}}{\mathrm{~d} t}=-J_{\gamma},
$$

where for the unperturbed problem $\mathbf{J}_{\gamma}=J_{\gamma} \hat{e}_{x}$ as given by Eq. 1, and we have used $\mathbf{J}_{\alpha}=\mathbf{J}_{\beta}=0$. After some algebra, we find

$$
\frac{\mathrm{d} x_{0}}{\mathrm{~d} t}=\frac{D_{\gamma} Q}{\left(c_{\gamma}^{\beta \gamma}-c_{\beta}^{\beta \gamma}\right)\left(e^{Q x_{1}}-e^{Q x_{0}}\right)}\left[c_{\gamma}^{\beta \gamma} e^{Q x_{1}}-c_{\gamma}^{\gamma \alpha} e^{Q x_{0}}\right]
$$

and

$$
\frac{\mathrm{d} x_{1}}{\mathrm{~d} t}=-\frac{D_{\gamma} Q}{\left(c_{\alpha}^{\gamma \alpha}-c_{\gamma}^{\gamma \alpha}\right)\left(e^{Q x_{1}}-e^{Q x_{0}}\right)}\left[c_{\gamma}^{\beta \gamma} e^{Q x_{1}}-c_{\gamma}^{\gamma \alpha} e^{Q x_{0}}\right] .
$$

These flux balances are consistent with the formulation of Shatynski et al. for the growth of a single intermetallic product phase formed from two phases with limited terminal solubility. ${ }^{13}$ In the "Results: Unperturbed Problem" section, we present results for how the motion of the unperturbed interfaces depends on system parameters and especially the 
magnitude and direction of the applied current through the parameter $Q$.

\section{Perturbed Problem}

We consider perturbations of the planar interfaces of the form $x_{0}^{(1)}(y, t)=x_{0}^{(0)}(t)+\epsilon_{0}(t) \cos \omega_{0} y$ and $x_{1}^{(1)}(y, t)=x_{1}^{(0)}(t)+\epsilon_{1}(t) \cos \omega_{1} y$, where $x_{0}(t)$ and $x_{1}(t)$ are the unperturbed (planar) solutions, $\epsilon_{0}(t)$ and $\epsilon_{1}(t)$ are the perturbation amplitudes and $\omega_{0}$ and $\omega_{1}$ are the perturbation frequencies. Our goal is to determine whether perturbations grow or decay by calculating the time rate of change of $\epsilon_{0}$ and $\epsilon_{1}$ under different electromigration conditions.

We assume the magnitudes of $\epsilon_{0}$ and $\epsilon_{1}$ are small compared with some characteristic length scale of the system (e.g., the initial thickness of the $\gamma$ phase) so that terms of order $\epsilon^{2}$ and higher may be neglected. Accordingly, we seek solutions of the form

$$
\phi(x, y ; t)=\phi^{(0)}(x ; t)+\phi^{(1)}(x, y ; t)
$$

and

$$
c(x, y ; t)=c^{(0)}(x ; t)+c^{(1)}(x, y ; t),
$$

where the perturbed solutions $\phi^{(1)}$ and $c^{(1)}$ have terms proportional to $\epsilon_{0} \cos \left(\omega_{0} y\right)$ and $\epsilon_{1} \cos \left(\omega_{1} y\right)$. Because we perform a linear stability analysis, we aim to derive sets of independent equations corresponding to the unperturbed solution and the two perturbations. As usual in this type of analysis, the unperturbed solution contributes to both the $\epsilon_{0}$ and $\epsilon_{1}$ solutions because we evaluate at the perturbed interfaces, though the $\epsilon_{0}$ and $\epsilon_{1}$ solutions do not interact with each other.

Consider first the electric potentials. The perturbed potentials $\phi^{(1)}$ for all three phases must satisfy Laplace's equation, and so it is straightforward to show that the potential in the $\gamma$ phase is

$$
\begin{aligned}
\phi_{\gamma}= & \phi_{\gamma}^{(0)}(x)+\epsilon_{0} a_{\gamma}\left[C_{1}^{\gamma} e^{\omega_{0} x}+C_{2}^{\gamma} e^{-\omega_{0} x}\right] \cos \left(\omega_{0} y\right) \\
& +\epsilon_{1} a_{\gamma}\left[C_{3}^{\gamma} e^{-\omega_{1} x}+C_{4}^{\gamma} e^{\omega_{1} x}\right] \cos \left(\omega_{1} y\right),
\end{aligned}
$$

where we have suppressed the time variable and we have explicitly shown the linear dependence on the unperturbed field strength $a_{\gamma}$. The potentials for the $\alpha$ and $\beta$ phases have the same form but with different constants $C_{1}-C_{4}$. The constants are found from the boundary and far-field conditions given by Eqs. 3-5. The boundary conditions are evaluated at the perturbed interfaces with normals $\hat{n}_{0}=$ $\hat{e}_{x}-\epsilon_{0} \cos \left(\omega_{0} y\right) \hat{e}_{y}$ and $\hat{n}_{1}=\hat{e}_{x}-\epsilon_{1} \cos \left(\omega_{1} y\right) \hat{e}_{y}$ to first order in $\epsilon$. After evaluating the $\phi^{(0)}$ at either $x=x_{0}+\epsilon_{0} \cos \left(\omega_{0} y\right) \quad$ or $\quad x=x_{1}+\epsilon_{1} \cos \left(\omega_{1} y\right) \quad$ and expanding to first order in $\epsilon$, we find six equations for the $\epsilon_{0}$ terms (two at each interface plus two farfield) and six for the $\epsilon_{1}$ terms. The solution of these equations give the 12 constants $C_{1}^{i}-C_{4}^{i}, i=\alpha, \beta, \gamma$.

Because we assume quasistatic diffusion, the composition field associated with the perturbed interface is found by ensuring that the electromigration-modified diffusion flux (1) is divergence free. As in the unperturbed case we only consider the composition in the $\gamma$ phase; however, we must consider the perturbations from both the interfaces at $x_{0}$ and $x_{1}$. That is, the perturbed composition $c^{(1)}$ is split up as $c^{(1)}=\epsilon_{0} c_{0}^{(1)}+\epsilon_{1} c_{1}^{(1)}$. By using Eqs. 14 and 9, we find that, to order $\epsilon_{1}$, the perturbed flux associated with the perturbation at the $\gamma / \alpha$ interface is

$$
\mathbf{J}_{1}^{(1)}=-D\left[\nabla c_{1}^{(1)}-Q \nabla c_{1}^{(1)}-\frac{Q}{a_{\gamma}} c^{(0)} \nabla \phi^{(1)}\right],
$$

and the composition $c_{1}^{(1)}$ satisfies

$$
\begin{aligned}
& \frac{\partial^{2} c_{1}^{(1)}}{\partial x^{2}}+\frac{\partial^{2} c_{1}^{(1)}}{\partial y^{2}}-Q \frac{\partial c_{1}^{(1)}}{\partial x} \\
& \quad=\omega_{1} Q^{2} \frac{c_{\gamma}^{\beta \gamma}-c_{\gamma}^{\gamma \alpha}}{e^{Q x_{1}}-e^{Q x_{0}}} e^{Q x}\left(C_{3}^{\gamma} e^{-\omega_{1} x}-C_{4}^{\gamma} e^{\omega_{1} x}\right)
\end{aligned}
$$

with similar equations for $\mathbf{J}_{0}^{(1)}$ and $c_{0}^{(1)}$. The solution to Eq. 18 is

$$
\begin{aligned}
c_{1}^{(1)}= & \epsilon_{1}\left\{A_{1} e^{\left(Q+\sqrt{Q^{2}+4 \omega_{1}^{2}}\right) x / 2}+B_{1} e^{\left(Q-\sqrt{Q^{2}+4 \omega_{1}^{2}}\right) x / 2}\right. \\
& \left.-Q \frac{c_{\gamma}^{\beta \gamma}-c_{\gamma}^{\gamma \alpha}}{e^{Q x_{1}}-e^{Q x_{0}}}\left(C_{3}^{\gamma} e^{\left(Q-\omega_{1}\right) x}+C_{4}^{\gamma} e^{\left(Q+\omega_{1}\right) x}\right)\right\} \\
& \times \cos \omega_{1} y .
\end{aligned}
$$

A similar result holds for $c_{0}^{(1)}$ :

$$
\begin{aligned}
c_{0}^{(1)}=\epsilon_{0}\{ & A_{0} e^{\left(Q+\sqrt{Q^{2}+4 \omega_{0}^{2}}\right) x / 2}+B_{0} e^{\left(Q-\sqrt{Q^{2}+4 \omega_{0}^{2}}\right) x / 2} \\
& \left.-Q \frac{c_{\gamma}^{\beta \gamma}-c_{\gamma}^{\gamma \alpha}}{e^{Q x_{1}}-e^{Q x_{0}}}\left(C_{1}^{\gamma} e^{\left(Q+\omega_{0}\right) x}+C_{2}^{\gamma} e^{\left(Q-\omega_{0}\right) x}\right)\right\}
\end{aligned}
$$

$\times \cos \omega_{0} y$.

The four new constants $A_{0}, A_{1}, B_{0}$, and $B_{1}$ are found from the boundary conditions at the $\beta / \gamma$ and $\gamma / \alpha$ interfaces. At the perturbed interfaces, we modify the equilibrium compositions $c_{\gamma}^{\beta \gamma}$ and $c_{\gamma}^{\gamma \alpha}$ by using the Gibbs-Thomsen boundary condition

$$
c=c^{0}(1+\Gamma \kappa)
$$

where $c^{0}$ is the equilibrium composition at a planar interface (i.e., the phase diagram composition used in the unperturbed problem), $\Gamma$ is a capillary constant proportional to the surface energy of the interface, and $\kappa$ is the mean curvature of the interface. ${ }^{14}$ For interfaces that are slightly perturbed from planar, $\kappa \approx-\nabla^{2} x$, so for the interfaces $x=x_{0}+\epsilon_{0} \cos \left(\omega_{0} y\right)$ and $x=x_{1}+\epsilon_{1} \cos \left(\omega_{1} y\right)$, the boundary conditions may be written

$$
\begin{aligned}
& c^{(0)}\left(x=x_{0}+\epsilon_{0} \cos \left(\omega_{0} y\right), y\right)+c_{0}^{(1)}\left(x_{0}, y\right) \\
& =c_{\gamma}^{\beta \gamma}\left(1+\Gamma \epsilon_{0} \omega_{0}^{2} \cos \left(\omega_{0} y\right)\right)
\end{aligned}
$$

and 


$$
\begin{aligned}
& c^{(0)}\left(x=x_{1}+\epsilon_{1} \cos \left(\omega_{1} y\right), y\right)+c_{1}^{(1)}\left(x_{1}, y\right) \\
& =c_{\gamma}^{\gamma \alpha}\left(1+\Gamma \epsilon_{1} \omega_{1}^{2} \cos \left(\omega_{1} y\right)\right),
\end{aligned}
$$

where for convenience we use the same capillary constant $\Gamma$ for both interfaces. By expanding $c^{(0)}$ to first order in $\epsilon_{0}$ and $\epsilon_{1}$ and matching orders, one can find a system of equations to solve for the constants $A_{0}, A_{1}$ and $B_{0}, B_{1}$.

Finally, we compute the velocity of the perturbed $\beta / \gamma$ and $\gamma / \alpha$ interfaces through the flux balances

$$
\left(c_{\gamma}^{\beta \gamma}-c_{\beta}^{\beta \gamma}\right) \frac{\mathrm{d} x}{\mathrm{~d} t}=\mathbf{J}_{\gamma} \cdot \hat{n}_{0} \quad \text { at } x=x_{0}(t)+\epsilon_{0}(t) \cos \left(\omega_{0} y\right)
$$

and

$$
\left(c_{\alpha}^{\gamma \alpha}-c_{\gamma}^{\gamma \alpha}\right) \frac{\mathrm{d} x}{\mathrm{~d} t}=-\mathbf{J}_{\gamma} \cdot \hat{n}_{1} \text { at } x=x_{1}(t)+\epsilon_{1}(t) \cos \left(\omega_{1} y\right),
$$

where $\mathbf{J}_{\gamma}$ is evaluated by using Eqs. 1, 9, 19, and 20 . After evaluating the terms and expanding to first order in $\epsilon_{0}$ and $\epsilon_{1}$, we find the speed of the unperturbed interfaces as in Eqs. 12 and 13, and the growth rate of the perturbations

$$
\begin{aligned}
\frac{1}{\epsilon_{0}} \frac{\mathrm{d} \epsilon_{0}}{\mathrm{~d} t}=\frac{\mathrm{d} \ln \epsilon_{0}}{\mathrm{~d} t} & \\
=\frac{1}{c_{\gamma}^{\beta \gamma}-c_{\beta}^{\beta \gamma}}[ & \lambda_{0}^{-} A_{0} e^{\lambda_{0}^{+} x_{0}}+\lambda_{0}^{+} B_{0} e^{\lambda_{0}^{-} x_{0}} \\
& +\mathcal{C} Q \omega_{0}\left(C_{1}^{\gamma} e^{\left(Q+\omega_{0}\right) x_{0}}-C_{2}^{\gamma} e^{\left(Q-\omega_{0}\right) x_{0}}\right) \\
& \left.+c_{\gamma}^{\beta \gamma} Q \omega_{0}\left(C_{1}^{\gamma} e^{\omega_{0} x_{0}}-C_{2}^{\gamma} e^{-\omega_{0} x_{0}}\right)\right]
\end{aligned}
$$

and

$$
\begin{aligned}
\frac{1}{\epsilon_{1}} \frac{\mathrm{d} \epsilon_{1}}{\mathrm{~d} t}=\frac{\mathrm{d} \ln \epsilon_{1}}{\mathrm{~d} t} & \\
=\frac{1}{c_{\alpha}^{\gamma \alpha}-c_{\gamma}^{\gamma \alpha}}[ & -\lambda_{1}^{-} A_{1} e^{\lambda_{1}^{+} x_{1}}-\lambda_{1}^{+} B_{1} e^{\lambda_{1}^{-} x_{1}} \\
& +\mathcal{C Q} \omega_{1}\left(C_{3}^{\gamma} e^{\left(Q-\omega_{1}\right) x_{1}}-C_{4}^{\gamma} e^{\left(Q+\omega_{1}\right) x_{1}}\right) \\
& \left.+c_{\gamma}^{\gamma \alpha} Q \omega_{1}\left(C_{3}^{\gamma} e^{-\omega_{1} x_{1}}-C_{4}^{\gamma} e^{\left(\omega_{1} x_{1}\right.}\right)\right],
\end{aligned}
$$

where in addition to the constants defined above, we have

$$
\mathcal{C}=\frac{c_{\gamma}^{\beta \gamma}-c_{\gamma}^{\gamma \alpha}}{e^{Q x_{1}}-e^{Q x_{0}}}
$$

and

$$
\lambda_{(0,1)}^{ \pm}=Q \pm \sqrt{Q^{2}+4 \omega_{(0,1)}^{2}} .
$$

\section{RESULTS}

We now consider how electric current affects both the motion of the planar interfaces between the phases as well as any perturbations of those interfaces. As mentioned in the "Introduction," results for the unperturbed problem have also been presented by Chen and Chen ${ }^{4-6}$ and Orchard and Greer. ${ }^{12,15}$

\section{Unperturbed Problem}

We integrate Eqs. 12 and 13 to compute the growth of the unperturbed $\gamma$ phase. We compute the thickness $t_{\gamma}=x_{1}-x_{0}$ of the $\gamma$ phase for different parameters. These parameters are normalized using a length scale $L_{0}$, chosen below to be the initial thickness of the $\gamma$ phase, and a time scale $T_{0}=L_{0}^{2} / D$. Compositions are scaled by the difference $c_{\beta}^{\beta \gamma}-c_{\alpha}^{\gamma \alpha}$. The normalization is straightforward and so in the following we do not distinguish between dimensionless and dimensional quantities.

We use the (now dimensionless) parameter $Q$ as the control parameter. From Eqs. 6 and 8 we see that $Q$ is proportional to the voltage drop and so can be varied independently for any choice of system parameters. The conductivity ratios $\delta_{\alpha}$ and $\delta_{\beta}$ affect the unperturbed solution only through their role in $Q$. The dependence of $Q$ on $x_{0}(t)$ and $x_{1}(t)$ is negligible when $L_{\alpha}$ and $L_{\beta}$ are large compared with $L_{0}$ and so will not be considered.

Figure 2 shows $t_{\gamma}$ for different values of $Q$. Other parameters are $\delta_{\alpha}=\delta_{\beta}=1, L_{\alpha}=L_{\beta}=500, c_{\gamma}^{\beta \gamma}=$ 0.42 , and $c_{\gamma}^{\gamma \alpha}=0.58$. We observe that, when $Q>0$, the growth rate of $\gamma$ is enhanced. On the other hand, when $Q<0$, the growth rate of $\gamma$ is retarded. These observations hold for all values of the system parameters, and are consistent with the observation that, when $Q>0$, electromigration drives flux in the same direction as the concentration gradient, i.e., from $\beta$ to $\alpha$.

The result coupling the direction of the current to an enhanced or diminished growth rate of the intermediate $\gamma$ phase is independent of the other system parameters. However, these parameters affect the growth of the intermediate phase in the absence of current, and also alter the magnitude of

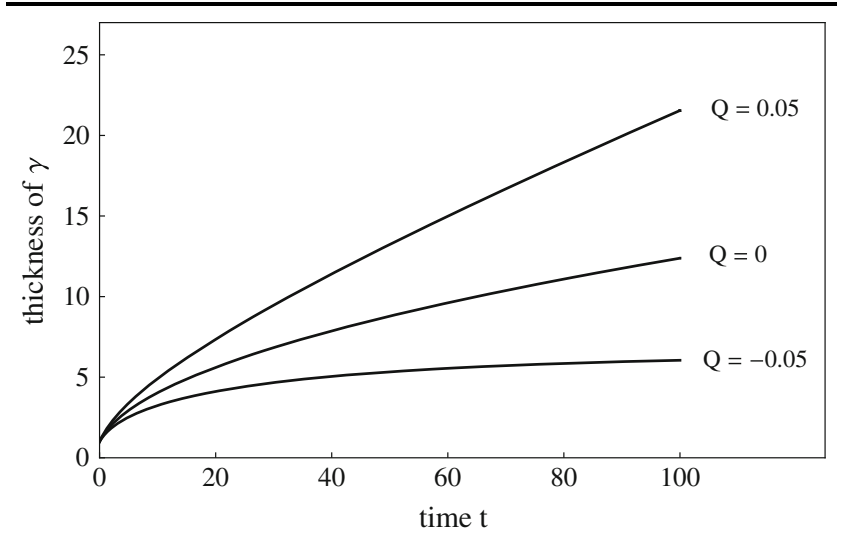

Fig. 2. Thickness of the intermediate $\gamma$ phase versus time for $\delta_{\alpha}=\delta_{\beta}=1$ and different values of $Q$. Other parameters are $L_{\alpha}=L_{\beta}=500, c_{\gamma}^{\beta \gamma}=0.58$, and $c_{\gamma}^{\gamma \alpha}=0.42$. The initial thickness of the $\gamma$ phase is 1 . 
the electromigration effect. Phase diagram compositions influence the growth of the $\gamma$ phase as seen in Eqs. 12 and 13. Note that in Fig. 2 we chose the composition of the intermediate phase to be centered at 0.5. Moving the center of the intermediate phase to either higher or lower compositions increases the electromigration effect. Also, decreasing the miscibility gap of the $\gamma$ phase region lowers the composition gradient across $\gamma$ and so slows its growth, while increasing that gap enhances its growth (figures not shown).

\section{Perturbed Problem}

The morphological stability of the $\beta / \gamma$ and $\gamma / \alpha$ interfaces is determined by the signs of $\mathrm{d} \ln \epsilon_{0} / \mathrm{d} t$ and $\mathrm{d} \ln \epsilon_{1} / \mathrm{d} t$ in Eqs. 22 and 23, respectively. If these growth rates are positive the interface is unstable with respect to the perturbations, while if they are negative the interface is stable. As we know that surface energy will stabilize the interfaces, we focus primarily on the case with zero surface energy, $\Gamma=0$, to see if there are any conditions under which electric current will destabilize the interface.

Consider first the baseline case where the system is electrically homogeneous, $\delta_{\alpha}=\delta_{\beta}=1$. In this case the perturbation has no effect on the electric field, which remains uniform over the diffusion couple. The current can still affect the morphological stability through its role in the unperturbed problem-note for example that $Q$ appears in the left-hand side of Eq. 18 for $c^{(1)}$ even though the right-hand side vanishes when $\delta_{\alpha}=\delta_{\beta}=1$. However, this effect is always stabilizing, that is, both $\mathrm{d} \ln \epsilon_{0} / \mathrm{d} t$ and $\mathrm{d} \ln \epsilon_{1} / \mathrm{d} t$ are less than zero for all values of $Q$. We also find that the diffusion problem in the absence of electric current $(Q=0)$ leads to morphologically stable interfaces.

The situation is more interesting when the conductivities of the phases differ. We focus first on the $\beta / \gamma\left(x_{0}\right)$ interface. Consistent with the linear analysis, we find that the conductivity ratio $\delta_{\alpha}$ does not affect the behavior of the $x_{0}$ interface and so is set to 1. (Similarly, the ratio $\delta_{\beta}$ does not affect the behavior of the $\gamma / \alpha$ interface.) Figure 3 shows $\mathrm{d} \ln \epsilon_{0} / \mathrm{d} t$ plotted against $Q$ when $\delta_{\beta}$ is 0.25 and 4 . The thickness $t_{\gamma}$ of the intermediate phase is 20 . When $\delta_{\beta}=0.25$, so the conductivity of the $\beta$ phase is less than that of the $\gamma$ phase, $\mathrm{d} \ln \epsilon_{0} / \mathrm{d} t$ is positive for large enough positive $Q$, and is negative for negative $Q$; that is, when $Q<0$, the $x_{0}$ interface is stabilized by current, while for large enough positive $Q$, the interface is destabilized by current. In contrast, when $\delta_{\beta}=4$ (also shown in Fig. 3), current destabilizes the interface when $Q$ is negative (less than about -0.05 ), while current stabilizes the interface when $Q$ is positive.

The results are similar at the $\gamma / \alpha\left(x_{1}\right)$ interface. Figure 4 shows $\mathrm{d} \ln \epsilon_{1} / \mathrm{d} t$ plotted against $Q$ when $\delta_{\beta}=1$ and $\delta_{\alpha}=0.25$ and 4 . Again, the sign of

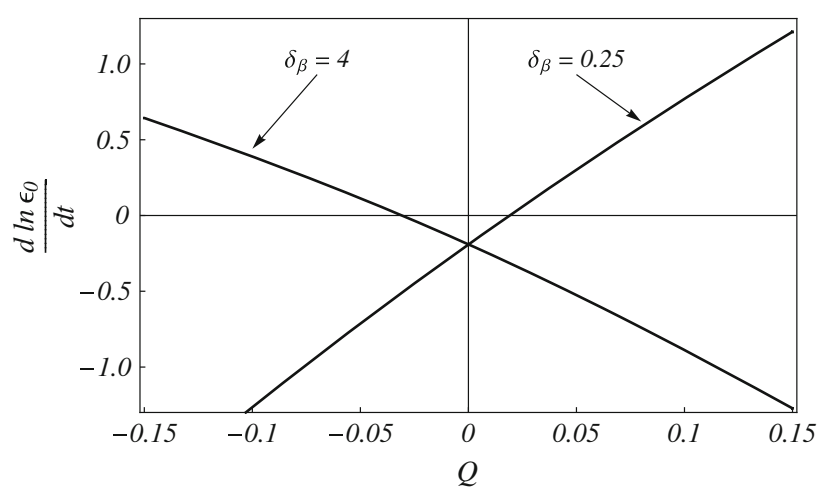

Fig. 3. Growth rate $\mathrm{d} \ln \epsilon_{0} / \mathrm{d} t$ of the perturbation at the $\beta / \gamma$ interface as a function of $Q$ for $\delta_{\beta}=0.25$ and $\delta_{\beta}=4$. Other parameters are $\Gamma=0, \quad \delta_{\alpha}=1, \quad t_{\gamma}=20, \quad \omega_{0}=10, \quad L_{\alpha}=L_{\beta}=500, \quad c_{\gamma}^{\beta \gamma}=0.58$, and $c_{\gamma}^{\gamma \alpha}=0.42$.

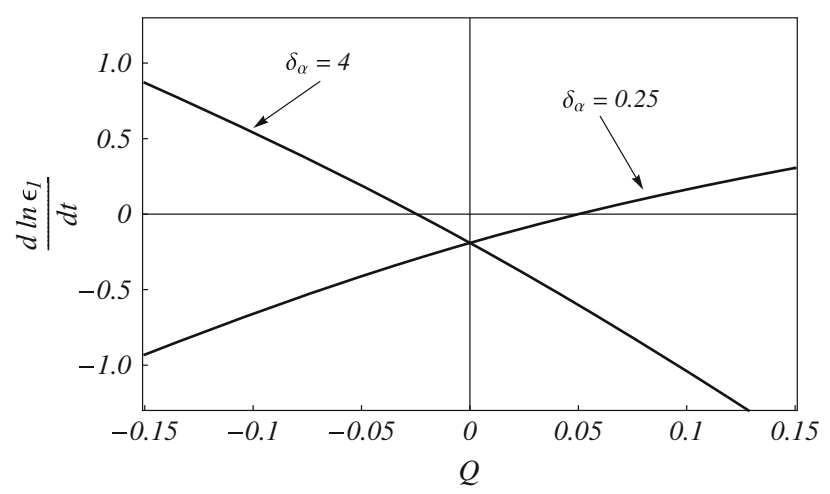

Fig. 4. Growth rate $\mathrm{d} \ln \epsilon_{1} / \mathrm{d} t$ of the perturbation at the $\gamma / \alpha$ interface as a function of $Q$ for $\delta_{\alpha}=0.25$ and $\delta_{\alpha}=4$. Other parameters are $\Gamma=0, \quad \delta_{\beta}=1, \quad t_{\gamma}=20, \quad \omega_{1}=10, \quad L_{\alpha}=L_{\beta}=500, \quad c_{\gamma}^{\beta \gamma}=0.58$, and $c_{\gamma}^{\gamma \alpha}=0.42$.

$\mathrm{d} \ln \epsilon_{1} / \mathrm{d} t$ depends on both $Q$ and $\delta_{\alpha}$. When $\delta_{\alpha}=0.25$, $\mathrm{d} \ln \epsilon_{1} / \mathrm{d} t$ is positive for large enough positive $Q$. When $\delta_{\alpha}=4, \mathrm{~d} \ln \epsilon_{1} / \mathrm{d} t$ is positive for large enough negative $Q$.

We conclude that electric current can destabilize an interface for certain values of $Q$ and the conductivity ratios $\delta_{\alpha}$ and $\delta_{\beta}$. The details depend on these parameters as well as the thickness $t$ of the intermediate phase. Figure 5 shows a stability diagram for the $\beta / \gamma$ interface as a function of $Q$ and $\delta_{\beta}$ with $t_{\gamma}=20$, while Fig. 6 shows a stability diagram for the same interface as a function of $Q$ and $t_{\gamma}$ with $\delta_{\beta}=0.25$ (so $Q>0$ is needed for instability). The corresponding figures for the $\gamma / \alpha$ interface are very similar. We see that, as the electrical contrast increases $\left(\delta_{\beta}\right.$ moves away from 1$)$, the magnitude of the current (in the appropriate direction) needed to destabilize the interface decreases. Also, as $t_{\gamma}$ increases, the magnitude of current required to drive an instability decreases. Alternatively, for a given current, there is some critical thickness below which the interface is stable. This is especially relevant when $\delta>1$, as this case requires negative $Q$ to drive the instability. Recall from the 


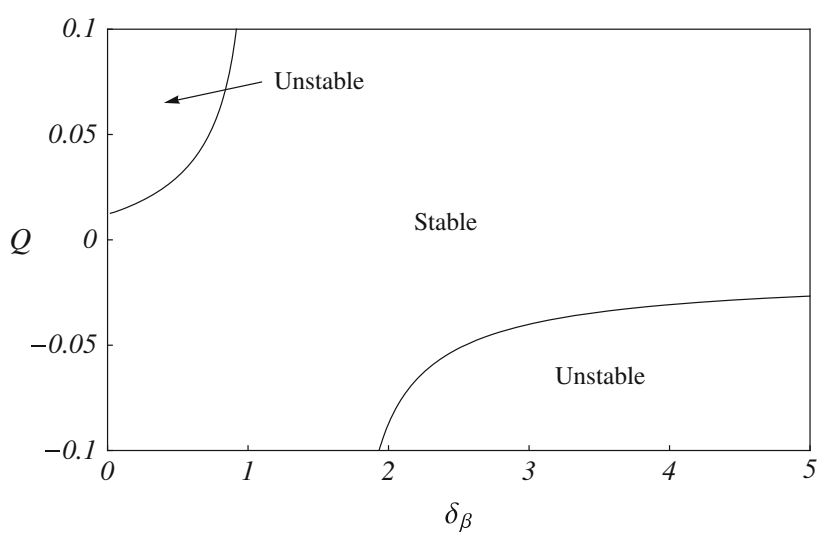

Fig. 5. Stability diagram showing the stability range of the $\beta / \gamma$ interface as a function of the parameters $Q$ and $\delta_{\beta}$. Other parameters are $\Gamma=0, \delta_{\alpha}=1, \quad t_{\gamma}=20, \omega_{0}=10, L_{\alpha}=L_{\beta}=500, \quad c_{\gamma}^{\beta \gamma}=0.58$, and $c_{\gamma}^{\gamma \alpha}=0.42$.

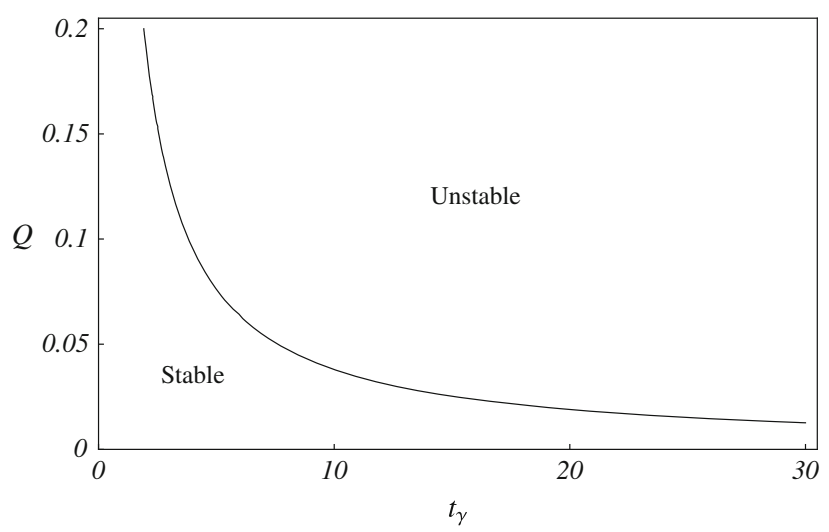

Fig. 6. Stability diagram showing the stability range of the $\beta / \gamma$ interface as a function of the parameters $Q$ and $t_{\gamma}$. Other parameters are $\Gamma=0, \delta_{\alpha}=1, \delta_{\beta}=0.25, \omega_{0}=10, L_{\alpha}=L_{\beta}=500, c_{\gamma}^{\beta \gamma}=0.58$, and $c_{\gamma}^{\gamma \alpha}=0.42$.

unperturbed problem that negative $Q$ slows the baseline growth of the intermediate phase. Hence trying to drive an instability by increasing the magnitude of $Q<0$ could be counterproductive, as it would also limit the growth of $t_{\gamma}$.

The remaining parameters-the values of the phase diagram compositions, the instability wavelengths, and the capillary constant $\Gamma$ - do not change the basic requirements for instability, though they do affect the stability boundaries. Phase diagram parameters can be reduced to the average composition $\left(c_{\gamma}^{\beta \gamma}+c_{\gamma}^{\gamma \alpha}\right) / 2$ and width $c_{\gamma}^{\gamma \alpha}-c_{\gamma}^{\beta \gamma}$ of the intermediate $\gamma$. Recall that $c$ gives the composition of the diffusing species B. We find that, as the $\gamma$ phase becomes $\mathrm{B}$ rich (and so the average composition increases), it becomes somewhat easier for current to destabilize the $x_{0}$ interface, though there is little effect on the $x_{1}$ interface. Similarly, conditions for instability are at best weakly dependent on the width $c_{\gamma}^{\gamma \alpha}-c_{\gamma}^{\beta \gamma}$ of the $\gamma$ phase region. The roles of the perturbation frequency and surface tension are straightforward. In the absence of surface tension, the magnitude of $\mathrm{d} \ln \epsilon / \mathrm{d} t$ increases linearly with $\omega$ at both the $\beta / \gamma$ and $\gamma / \alpha$ interfaces. In contrast, the surface energy contribution scales as $\omega^{3}$. Hence, as expected, surface energy stabilizes high-frequency perturbations and would give rise to a fastest-growing wavelength when current is destabilizing.

\section{DISCUSSION}

It is well known that electric current can either increase or decrease solute diffusion through the electromigration term in the diffusion flux (1). This in turn can enhance or retard the growth of intermediate phases in binary diffusion couples. ${ }^{4}$ Electromigration enhances intermediate phase growth when current drives solute flux from the high-solute phase towards the low-solute phase $(Q>0$ in our notation), and slows growth in the alternative case.

Current can in some cases also drive a morphological instability of the interfaces between the intermediate and the terminal phases. As shown in the results, the conditions for instability depend on the sign of $Q$ and the conductivity ratios $\delta_{\alpha}$ and $\delta_{\beta}$. The reason is as follows. It is straightforward to show that the most significant contributor to the perturbed flux in Eq. 17 is the term associated with the perturbed electric field $\nabla \phi^{(1)}$. At the $\beta / \gamma$ interface this term is proportional to $Q\left(\delta_{\beta}-1\right) \cos \omega_{0} y$, while at the $\gamma / \alpha$ interface it is proportional to $-Q\left(\delta_{\alpha}-1\right) \cos \omega_{1} y$. Consider first the $\gamma / \alpha$ interface. If $Q>0$ and $\delta_{\alpha}>1$, solute flux is reduced at the peaks of the perturbation $\left(\cos \omega_{1} y=1\right)$ and enhanced at the valleys. Because this interface moves via solute incorporation (to the right as in Fig. 1), this means the valleys grow relative to the peaks and the interface is stabilized. Alternatively, if $Q>0$ and $\delta_{\alpha}<1$, solute flux is enhanced at the peaks and reduced at the valleys and so the interface is destabilized. A similar situation arises at the $\beta / \gamma$ interface, though in this case the interface moves to the left (towards $\beta$ ) via solute rejection. Hence, if $Q>0$ and $\delta_{\beta}>1$, solute flux is enhanced at peaks $\left(\cos \omega_{0} y=1\right)$ and reduced at the valleys. This causes the peaks to move left faster than the valleys, which reduces the perturbation amplitude and so stabilizes the interface.

We can qualitatively compare our results with the experimental observations of Chen and Chen. ${ }^{4-6}$ Chen and Chen ran a series of experiments on the role of current in the growth of intermetallics in several different binary diffusion couples, including $\mathrm{Sn} / \mathrm{Ag}$ and $\mathrm{Sn} / \mathrm{Ni}$. In both systems, $\mathrm{Sn}$ is the primary diffusing species, and so Sn would be the $\beta$ phase and $\mathrm{Ag}$ (or $\mathrm{Ni}$ ) the $\alpha$ phase in our notation. These couples were allowed to evolve at moderate temperatures in the absence of current, such that an intermetallic phase $\left(\mathrm{Ag}_{3} \mathrm{Sn}\right.$ or $\left.\mathrm{Ni}_{3} \mathrm{Sn}_{4}\right)$ grew at the two-phase interface over the course of several 
hundred hours. Each couple was then retested under current, both from $\mathrm{Sn}$ to $\mathrm{Ag}$ and from $\mathrm{Ag}$ to $\mathrm{Sn}$, and the measured thicknesses of the intermetallic were compared with the thickness in the absence of current.

To compare their results with theory, Chen and Chen defined a parameter

$$
U_{\mathrm{C} / \mathrm{C}}=-\frac{D F}{R T} \frac{z^{*}}{f} E
$$

where $D$ is the diffusion constant of the diffusing species, $F$ is Faraday's constant, $R$ is the gas constant, $T$ is temperature, and $z^{*}$ and $f$ are the effective charge and correlation coefficient of the diffusing species. Also $E=\frac{I \rho}{A}$ is the electric field in the intermediate layer, with $I$ being the current, $\rho$ the resistivity of that layer, and $A$ its cross-sectional area. Since $E=-\nabla \phi=-a_{\gamma}$ for the unperturbed problem, we see from Eq. 8 that our $Q=\frac{U_{\mathrm{C} / \mathrm{C}}}{D}$, and so in particular has the same sign as $U_{\mathrm{C} / \mathrm{C}}$. As here, Chen and Chen showed that growth of the $\gamma$ phase is enhanced when $U_{\mathrm{C} / \mathrm{C}}>0$ and retarded when $U_{\mathrm{C} / \mathrm{C}}<0$. This agrees with their experimental observations on $\mathrm{Sn} / \mathrm{Ag}$ and $\mathrm{Sn} / \mathrm{Ni}$ diffusion couples. For example, in the Sn/Ag system, ${ }^{6}$ Chen and Chen find that, for a $5 \mathrm{~A} / \mathrm{mm}^{2}$ current density from $\mathrm{Sn}$ to $\mathrm{Ag}$ and at a temperature of $140^{\circ} \mathrm{C}$, the thickness of the intermetallic $\mathrm{Ag}_{3} \mathrm{Sn}$ phase is about $20 \%$ higher than in the absence of current. When the current direction is reversed, the thickness of the intermetallic is about $20 \%$ less than in the absence of current.

We compare our unperturbed results to Chen and Chen's results. Chen and Chen's micrographs consistently show enhanced growth of intermetallic with the label that the "flow direction of electrons" is from $\mathrm{Sn}$ to $\mathrm{Ag}(\mathrm{Ni})$; in the text they refer to the same situation as "electron current flow" from $\mathrm{Sn}$ to $\mathrm{Ag}$ (Ni). Based on this observation and the above discussion of the signs of $U_{\mathrm{C} / \mathrm{C}}$ and $Q$, we associate our $Q>0$ case with electron current flow from $\mathrm{Sn}$ to $\mathrm{Ag}$ (Ni), i.e., current flow to the right in Fig. 1.

For the $\mathrm{Sn} / \mathrm{Ag}$ system, Chen and Chen report phase diagram values $c_{\gamma}^{\beta \gamma}=0.237$ and $c_{\gamma}^{\gamma \alpha}=0.25$. Also, at $120^{\circ} \mathrm{C}$, the diffusivity of $\mathrm{Sn}$ in $\gamma$ is $D=$ $0.734 \mu \mathrm{m}^{2} / \mathrm{h}$, and the resistivity of $\gamma$ is $\rho=0.182$ $\Omega \mu \mathrm{m}$. From their experiments they infer a value of $U_{\mathrm{C} / \mathrm{C}}=0.00256 \mu \mathrm{m} / \mathrm{h}$ and a corresponding $\left|z^{*}\right|=120$ (the sign of $z^{*}$ is included in our discussion of the sign of $Q$ and the direction of current). This gives the dimensional $Q=34.5 / \mathrm{cm}$. If we take a length scale $\tilde{L}=1 \mu \mathrm{m}$, then the dimensionless $\tilde{Q}=0.00345$. Also the time scale $\tilde{T}=\tilde{L}^{2} / D=1.36 \mathrm{~h}$. These values are input into the differential equations (12) and (13) and solved to find the thickness of the interface as a function of time. As expected, we find excellent agreement with Chen and Chen's results, with only small quantitative differences owing to the fact that Chen and Chen only consider motion of the $\mathrm{Ag}_{3} \mathrm{Sn} / \mathrm{Ag}(\gamma / \alpha)$ interface.
It is more challenging to compare our results on the morphological stability of the $\mathrm{Sn} / \mathrm{Ag}_{3} \mathrm{Sn}(\beta / \gamma)$ and $\mathrm{Ag}_{3} \mathrm{Sn} / \mathrm{Ag}(\gamma / \alpha)$ interfaces with Chen and Chen's published micrographs. Using the resistivity of $\gamma$ as reported above, and values of $\rho=0.11 \Omega \mu \mathrm{m}$ for tin $(\beta)$ and $0.0147 \Omega \mu \mathrm{m}$ for silver $(\alpha),{ }^{16}$ we calculate $\delta_{\alpha}=12.4$ and $\delta_{\beta}=1.7$. We also take $t_{\gamma}=10$, roughly consistent with Chen and Chen's micrographs. We do not explicitly consider surface energy, though we remark that for $Q=-0.035$ a dimensional value of the capillary length of $\Gamma=10^{-7} \mathrm{~cm}$ (roughly a surface energy of $1 \mathrm{~J} / \mathrm{m}^{2}$ ) effectively suppresses the instability for all but very long-wavelength instabilities.

For the parameters above, we find that electromigration can potentially drive instability of both the $\beta / \gamma$ and $\gamma / \alpha$ interfaces for large enough negative $Q$. Also the electromigration effect is stronger at the $\gamma / \alpha$ interface because the contrast in conductivities is larger across that interface. In contrast, in Chen and Chen's micrographs of the $\mathrm{Sn} / \mathrm{Ag}$ system, one observes small perturbations of the $\mathrm{Sn} / \mathrm{Ag}_{3} \mathrm{Sn}(\beta / \gamma)$ interfaces for both the cases $Q>0$ and $Q<0$. Also the $\mathrm{Ag}_{3} \mathrm{Sn} / \mathrm{Ag}(\gamma / \alpha)$ interface is planar independent of $Q$. Similar observations can be made in other cases, for example, in the $\mathrm{Sn} / \mathrm{Ni}$ system $^{6,5}$ (though the roughness of the $\mathrm{Sn} / \mathrm{Ni}_{3} \mathrm{Sn}_{4}$ interface in that case is much more random than in the $\mathrm{Sn} / \mathrm{Ag}$ case). Also we do not find any conditions in our analysis under which the same interface (i.e., the $\mathrm{Ag}_{3} \mathrm{Sn} / \mathrm{Sn}$ interface) is unstable for both $Q>0$ and $Q<0$. We conclude that the roughness of the interfaces in Chen and Chen's micrographs is not the result of a morphological instability of the type considered here.

\section{CONCLUSIONS}

The role of electromigration in the morphological stability of an interface in a binary diffusion couple is determined by the direction of current (sign of $Q$ ) and the ratio of conductivities on either side of the interface. Electromigration can drive a morphological instability during the growth of an intermediate phase in a binary diffusion couple. Instability of an interface requires that the sign of $Q(\delta-1)$ be negative, where $\delta$ is the ratio of the electrical conductivity of the terminal phase to that of the intermediate phase. Instability also requires that the thickness of the intermediate phase be larger than some critical value that depends on both the relative conductivities and the magnitude of the current. We expect that such conditions may be possible in practice. However, in many cases the conductivity of the growing intermediate phase is less than that of the surrounding pure metals. In this case $\delta>1$ and instability requires $Q<0$, which slows the overall growth of the intermediate phase. Hence, it may be difficult to grow the intermediate phase thick enough to observe instability experimentally in such systems. 


\section{REFERENCES}

1. H.B. Huntington, Diffusion in Solids, Recent Developments, ed. A.S. Nowick and J.J. Burton (New York: Academic, 1975), pp. 303-352.

2. K.N. Tu, J. Appl. Phys. 94, 5451 (2003).

3. L.E. Felton, A.D. Selsley, and P.J. Ficalbora, Appl. Phys. Lett. 54, 2074 (1989).

4. C.-M. Chen and S.-W. Chen, J. Electron. Mater. 28, 902 (1999).

5. C.-M. Chen and S.-W. Chen, J. Appl. Phys. 90, 1208 (2001).

6. C.-M. Chen and S.-W. Chen, Acta Mater. 50, 2461 (2002).

7. P. Decuzzi, Thin Solid Films 437, 188 (2003).

8. L. Klinger and L. Levin, J. Appl. Phys. 78, 1669 (1995).
9. L. Klinger, L. Levin, and D. Srolovitz, J. Appl. Phys. 79, 6834 (1996).

10. J. Cho, M.R. Gungor, and D. Maroudas, Appl. Phys. Lett. 85, 2214 (2004).

11. V. Tomar, M.R. Gungor, and D. Maroudas, Phys. Rev. Lett. 100, 036106 (2008).

12. H.T. Orchard and A.L. Greer, Appl. Phys. Lett. 86, 1 (2005).

13. S.R. Shatynski, J.P. Hirth, and R.A. Rapp, Acta. Metall. 24, 1071 (1976).

14. W.W. Mullins and R.F. Sekerka, J. Appl. Phys. 34, 323 (1963).

15. H.T. Orchard and A.L. Greer, J. Electron. Mater. 35, 1961 (2006).

16. Metals Handbook, 2nd ed. (Materials Park, OH: ASM International, 1998). 